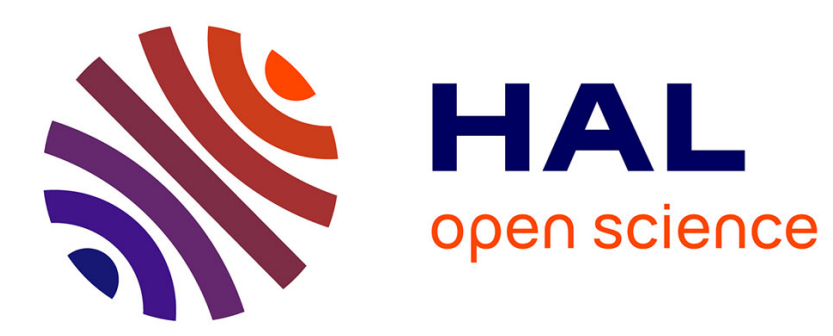

\title{
Finite Element simulation of buckling-induced vein tortuosity and influence of the wall constitutive properties
}

Pierre Badel, Christian Pierre-Yves Rohan, Stéphane Avril

\section{To cite this version:}

Pierre Badel, Christian Pierre-Yves Rohan, Stéphane Avril. Finite Element simulation of bucklinginduced vein tortuosity and influence of the wall constitutive properties. Journal of the mechanical behavior of biomedical materials, 2013, 26, pp. 119-126. 10.1016/j.jmbbm.2013.05.006 . hal-00879052

\section{HAL Id: hal-00879052 https://hal.science/hal-00879052}

Submitted on 5 Nov 2013

HAL is a multi-disciplinary open access archive for the deposit and dissemination of scientific research documents, whether they are published or not. The documents may come from teaching and research institutions in France or abroad, or from public or private research centers.
L'archive ouverte pluridisciplinaire HAL, est destinée au dépôt et à la diffusion de documents scientifiques de niveau recherche, publiés ou non, émanant des établissements d'enseignement et de recherche français ou étrangers, des laboratoires publics ou privés. 
Finite Element simulation of buckling-induced vein tortuosity and influence of the wall constitutive properties

Pierre BADEL a, Christian Pierre-Yves ROHAN a, Stéphane AVRIL a

a Ecole Nationale Supérieure des Mines de Saint Etienne, CIS-EMSE, CNRS:UMR5146, LCG, 158 cours Fauriel, 42023 Saint-Etienne Cedex 2, France

email adresses : \{badel, rohan, avril\}@emse.fr

\section{Corresponding author}

Pierre BADEL

Ecole Nationale Supérieure des Mines de Saint Etienne, 158 cours Fauriel, 42023 Saint-Etienne Cedex 2, France

Email : badel@emse.fr

Tel : $+33(0) 477420260$ 


\begin{abstract}
The mechanisms giving rise to vein tortuosity, which is often associated with varicosis, are poorly understood. Recent works suggest that significant biological changes in the wall of varicose veins may precede the mechanical aspects of the disease. To test the hypothesis of tortuosity being a consequence of these changes, a Finite Element model was developed based on previous experimental work on vein buckling. The model was then used to evaluate the effect of alterations of the mechanical behavior of the wall on tortuosity onset and severity. The results showed that increasing anisotropy towards the circumferential direction promotes tortuosity. An increase in wall stiffness tends to decrease the level of tortuosity but interestingly, if the vein segment is little or not pre-stretched such increase will not prevent, or it will even promote, the onset of tortuosity. These results provide additional arguments supporting the hypothesis of tortuosity being the consequence of biologically-induced changes in the varicose vein wall. Based on a 3D model of the leg and in vivo identification of the material properties of varicose veins, a clinical validation of these findings is being developed.
\end{abstract}




\section{Introduction}

Though it is less studied than the arterial system, the venous system may be subjected to disorders affecting the vascular function. Containing about three fourths of the peripheral blood volume (Attinger, 1969), the peripheral venous system has to maintain the blood return to the heart. This system can undergo disorders leading to pathological consequences such as heavy legs, oedema, phlebitis or pulmonary embolism. Another widespread venous disease is varicosis which is often associated with vein tortuosity (Caggiati et al., 2006).

The causes of vessel tortuosity are poorly understood. Most of the related studies have been performed from the clinical or biological point of view, quite often focusing on arterial tortuosity and associated diseases (Smedby and Bergstrand, 1996). Regarding the venous system, it was shown that several significant biological disorders occur in varicose veins, with structural alteration in matrix composition (Naoum et al., 2007; Woodside et al., 2003). Travers et al. (1996) observed that varicose saphenous veins contained significantly higher amounts of collagen in all layers of the vein wall and that these collagen fibers were seen to invade and break up regular muscle layers of the media in varicosis. Concurrently, smooth muscle cell synthetic capacity was found to be modified in varicose veins, which may contribute to the remodeling and weakening of the venous wall (Urbanek et al., 2004; Xiao et al., 2009). Though it was long thought that they were purely the consequences of mechanical factors like venous hypertension and valvular incompetence, these biological changes are now seen to be central to varicose vein development. Recent evidence suggests that they may precede valvular dysfunction (Lim and Davies, 2009). These findings demonstrate that mechanical factors may not be the leading cause triggering venous wall disorders. They may be only a consequence of it, and modulate the severity of the disease (Oklu et al., 2012).

For these reasons, there is a pressing need to investigate the hypothesis that the formation of venous tortuosity is a mechanical consequence of vein wall degradation. This kind of hypothesis was already formulated for arterial tissue and is still the object of research focusing on the mechanical effects of arterial wall disorders and remodeling (Carta et al., 2009; Dye et al., 2007; Wagenseil et al., 2005). The biomechanical aspects of vessel tortuosity have been addressed through a considerable amount of work by the group of H-C. Han which focused on the mechanism of vessel buckling, both on arteries and veins. Most of this work is analytical and strongly supports the hypothesis of tortuosity being triggered by mechanical buckling (Han, 2008; Lee and Han, 2010). Experiments were also performed which confirm these findings in in 
vitro conditions (Liu and Han, 2012; Martinez et al., 2010). This impressive work clearly demonstrated the relevance of the hypothesis of buckling as a mechanism responsible for vessel tortuosity. However, the aspects related to possible alterations of the vessel's mechanical behavior and its effects were not addressed by these authors. Yet, the experimental study by McGilvray et al. (2010) who studied post-phlebitic conditions in rat models brought clear evidence that such disorders lead to altered mechanical properties.

Based on these findings, our goal is to introduce a numerical Finite Element (FE) model able to reproduce the phenomenon of tortuosity forming via a buckling mechanism and study the consequence of vein wall degradation on this mechanism. The objective of the present paper is to show the feasibility of a simple and efficient FE model demonstrating the mechanism of buckling-induced venous tortuosity, and use it to evaluate how changes in the mechanical behavior of the venous wall would affect tortuosity.

\section{Methods}

In order to study buckling-induced tortuosity in pressurized veins and test the influence of the wall constitutive parameters on this phenomenon, a numerical FE model of a single vein segment was created. The model characteristics were based on the experiments performed by Martinez et al. (2010) on jugular veins of 6 to 7-month-old pigs for defining the geometry and boundary conditions, identifying the constitutive properties, and validating the model. This model was further used to test various scenarii of constitutive properties evolution.

\subsection{Finite Element Model description}

The geometry of the model was based on the experimental buckling tests developed by Martinez et al. (2010). Using mid-range values reported in this paper, the FE model of the vein segment was defined as a $30 \mathrm{~mm}$ long, $6.5 \mathrm{~mm}$ diameter and $0.3 \mathrm{~mm}$ thick cylindrical segment. This geometry was discretized with a regular mesh of 2400 four-node reduced integration shell elements (S4R in Abaqus $囚$ ), resulting in 14400 degrees of freedom.

Similarly to the experiment mentioned above, displacement boundary conditions were applied onto the nodes belonging to each end section of the segment. One end was fixed by blocking all the translational degrees of freedom of the corresponding nodes. The other end had its nodes blocked in the radial direction, while an axial displacement was imposed to achieve the desired axial pre-stretch, defined here as the ratio of the vessel length to its initial unloaded length, and ranging from 1 to 1.6. This pre-stretch is present in vivo when the vessel is not pressurized, and it can span a wide range of values as can be found in the literature for various types of arterial and venous vessels (Brossollet and Vito, 1995; Guo et al., 2012). After the desired pre-stretch 
was reached, a smoothly and gradually increasing internal pressure was applied onto the inner surface of the segment up to $100 \mathrm{mmHg}$.

In order to simulate buckling and tortuosity onset of the segment in a simple way, and to allow for possible future use in a more complex model (surrounding tissue and leg geometry, for instance), the problem was solved with an explicit scheme. The advantage of this type of solver is that it is very robust in non-linear and unstable problems, hence suitable in buckling simulation. However, in order to keep the problem in quasi-static conditions and have a numerically and physically meaningful solution, it is important that the ratio of the kinetic energy of the system to the total internal energy (here, strain energy) is maintained below $5 \%$ (Kim et al., 2002). Otherwise, inertia forces will dominate. In the simulations presented here, this ratio was always much below $5 \%$ except during the few increments when buckling initiated (in this situation, the ratio was found to reach up to $30 \%$ ).

\subsection{Constitutive properties}

The experimental data from the inflation-extension tests of Martinez et al. (2010) were fitted with a Fung-type constitutive model in a subsequent study by the same team (Lee and Han, 2010). In the present paper, it was decided to use the anisotropic hyperelastic constitutive model introduced by Holzapfel et al. (2000) with a single layer, two symmetrically-oriented fiber families and the assumption of incompressibility which is common in soft tissue modeling. This model was preferred for its numerical stability, especially at low strains (Holzapfel et al. 2004), which is important for the sensitivity analysis tests performed in the following. In addition, the parameters of this model are structurally motivated. The strain energy function of this model can be written in the following form:

$$
\psi=\frac{\mathrm{C}_{10}}{2}\left(\mathrm{I}_{1}-3\right)+\sum_{\mathrm{i}=4,6} \frac{\mathrm{k}_{1}}{2 \mathrm{k}_{2}}\left[\exp \left(\mathrm{k}_{2}\left(\mathrm{I}_{\mathrm{i}}-1\right)^{2}\right)-1\right]
$$

where, $\mathrm{C}_{10}$ is the parameter of the isotropic neo-Hookean term, $\mathrm{k}_{1}$ and $\mathrm{k}_{2}$ are the parameters for the exponential response of the fiber families, and the structural anisotropy induced by the fiber network arises from both $\mathrm{I}_{4}$ and $\mathrm{I}_{6}$. These terms are pseudo-invariants of the right Cauchy Green tensor $\underline{\underline{\mathbf{C}}}$ and the fiber directions $\underline{\mathbf{f}}_{1}$ and $\underline{\mathbf{f}}_{2}$. They are driven by the parameter $\beta$, the mean fiber angle in the medium, defined in Fig. 1. They can be expressed in terms of the squares of stretch of the two fiber families, $\mathrm{I}_{4}=\underline{\mathbf{f}} \underline{\mathbf{f}}_{1} . \underline{\underline{\mathbf{C}}} \underline{\underline{\mathbf{f}_{1}}}=\lambda_{\text {fiber } 1}^{2}$ and $\mathrm{I}_{6}=\underline{\mathbf{f}}_{2} . \underline{\underline{\mathbf{C}}}_{\underline{\mathbf{f}}} \underline{\underline{f}}_{2}=\lambda_{\text {fiber } 2}^{2}$. For completeness, we recall here the analytical expressions of the circumferential and axial Cauchy stresses in a cylindrical basis, derived from Eq. (1) assuming that the fiber directions are symmetric with respect to the circumferential direction: 


$$
\begin{aligned}
& \sigma_{\theta \theta}^{\text {model }}=\mathrm{C}_{10}\left(1+2 \mathrm{E}_{\theta \theta}\right)+4 \mathrm{k}_{1} \cos ^{2} \beta\left(\mathrm{I}_{4}-1\right) \exp \left[\mathrm{k}_{2}\left(\mathrm{I}_{4}-1\right)^{2}\right]\left(1+2 \mathrm{E}_{\theta \theta}\right) \\
& \sigma_{\mathrm{zz}}^{\text {model }}=\mathrm{C}_{10}\left(1+2 \mathrm{E}_{\mathrm{zz}}\right)+4 \mathrm{k}_{1} \sin ^{2} \beta\left(\mathrm{I}_{4}-1\right) \exp \left[\mathrm{k}_{2}\left(\mathrm{I}_{4}-1\right)^{2}\right]\left(1+2 \mathrm{E}_{\mathrm{zz}}\right)
\end{aligned}
$$

where $\mathrm{I}_{4}-1=\mathrm{I}_{6}-1=2 \mathrm{E}_{\theta \theta} \cos ^{2} \beta+2 \mathrm{E}_{\mathrm{zz}} \sin ^{2} \beta$.

To identify the four parameters of this model, an inverse approach was set up based on a cost function and a minimization algorithm. The analytical cost function $\mathrm{J}$ was built upon the difference between experimental and modeled stress values at each recorded state of the vein segment:

$$
\mathrm{J}(\underline{\mathrm{p}})=\sum_{\mathrm{i}=1}^{\mathrm{n}}\left[\sigma_{\theta \theta}^{\text {model }}\left(\underline{\mathrm{p}}, \mathrm{E}_{\theta \theta}^{\mathrm{i}}, \mathrm{E}_{z z}^{\mathrm{i}}\right)-\sigma_{\theta \theta}^{\mathrm{exp}}\left(\mathrm{E}_{\theta \theta}^{\mathrm{i}}, \mathrm{E}_{z z}^{\mathrm{i}}\right)\right]^{2}+\left[\sigma_{z z}^{\text {model }}\left(\underline{\mathrm{p}}, \mathrm{E}_{\theta \theta}^{\mathrm{i}}, \mathrm{E}_{z z}^{\mathrm{i}}\right)-\sigma_{z z}^{\exp }\left(\mathrm{E}_{\theta \theta}^{\mathrm{i}}, \mathrm{E}_{z z}^{\mathrm{i}}\right)\right]^{2}
$$

where $\mathrm{p}$ is the vector of constitutive parameters to be identified. Superscripts "model" and "exp" respectively stand for the values obtained from the model detailed above (see Eq. 2) and the values obtained from the Fung-type model fitted by Lee and Han (2010) on their previous experimental data. $\mathrm{E}_{\theta \theta}^{\mathrm{i}}$ and $\mathrm{E}_{z z}^{\mathrm{i}}$ are the circumferential and axial Green-Lagrange strains at the $\mathrm{i}^{\text {th }}$ recorded state ( $\mathrm{n}$ being the number of recorded states).

This cost function was minimized with respect to the unknown constitutive parameters using the default algorithm implemented for non-linear least-square problems in Matlab®.

\subsection{Data collected}

For the purpose of validation, the critical buckling pressure was extracted from the FE model. To remain consistent with the buckling data used (Martinez et al., 2010), the critical pressure was defined as the minimal pressure at which the deflection of the vein segment exceeded $0.5 \mathrm{~mm}$. The deflection was computed from the deformed coordinates of the mesh as the maximum deviation of the centerline of the segment from the initial straight centerline.

To characterize tortuosity, we used the ratio of the deflection to the non-pressurized prestretched vessel length as a tortuosity index (TI) :

$$
\mathrm{TI}=\frac{\mathrm{d}}{\lambda \mathrm{L}_{0}}
$$

where $d$ is the maximum value of the centerline's deflection, $L_{0}$ the initial length of the model (30 $\mathrm{mm}$ ), and $\lambda$ the axial pre-stretch imposed.

\subsection{Scenarii of constitutive property evolution}

After validation against the experimental critical pressures, the FE model presented above was used to study two scenarii in which the vein's constitutive behavior is altered. The purpose was 
to determine their influence on the critical pressure and tortuosity, and give some clues on the possible mechanisms leading to varicose veins in vivo.

First, the influence of anisotropy was studied by varying the fiber angle $\beta$ in the range $15-45^{\circ}$, the other parameters being maintained at the value identified previously. Note that $\beta=45^{\circ}$ corresponds to an isotropic-like tissue, in the sense that its initial (low-strain) response is the same in circumferential and axial directions while small values of $\beta$ represent an anisotropic tissue, with the circumferential direction being stiffer. To support the choice of this range, evidences were found in the literature showing that venous tissues are markedly anisotropic with the circumferential direction being stiffer (Martinez et al., 2010; McGilvray et al., 2010; Weizsäcker, 1988) and preliminary simulations showed that no buckling appeared with $\beta>45^{\circ}$ and buckling was instantaneous or almost instantaneous with $\beta<15^{\circ}$ making this range of value not relevant for the present study.

Second, the influence of stiffness was studied by simultaneously scaling both the parameters $\mathrm{C}_{10}$ and $\mathrm{k}_{1}$ by factors ranging from 0.1 to 10 , the other parameters being maintained at the value identified previously. This scenario was inspired from the results of McGilvray et al. (2010) who found marked changes in the values of these parameters in altered venous walls compared to normal ones.

\section{Results}

\subsection{Identification and validation}

The identification procedure was run in Matlab $₫$ and provided the parameters reported in Table 1. Using these values, we obtained an excellent fit of the inflation-extension data, with RMSE $=2.2 \mathrm{kPa}$, while stresses range between up to $300 \mathrm{kPa}$ (curves not shown here). We shall refer to the calibrated model as the reference model for the rest of the present paper.

As a validation step, the reference model was used to numerically reproduce the buckling experiments reported by Martinez et al. (2010). An example of the response of this model is shown in Fig. 2 where the tortuosity index is plotted as a function of the pressure for different axial pre-stretches. A visual illustration of the results is also provided. Fig. 3 shows the results obtained in terms of critical pressure as a function of the imposed axial pre-stretch, compared to the mean values reported experimentally. Out of the range 1.2 to 1.5 of axial pre-stretch, the model over-estimates the critical pressure by 10 to $20 \mathrm{mmHg}$. In the range 1.2 to 1.5 of axial prestretch, the model correctly predicts the effect of the axial stretch on the buckling pressure. However, deviations between the buckling pressures exist between the experiments and the simulations, they may be explained by the possible local defects existing in the real vein, and which are prone to induce premature buckling. These remarks lead us to consider that the 
model is acceptable for the objectives of this study in the sense that it captures the main trends regarding the buckling response of venous segments.

To complete these results, the authors evaluated the in vivo axial pre-stretch of this model vein segment using the method suggested in Brossollet and Vito (1995) and inspired from previous experiments (Humphrey et al., 1993; Van Loon et al., 1977; Weizsacker and Pinto, 1988). This method consists in plotting the mechanical response to pressurization in terms of axial force (F) vs. pressure ( $p$ ) for different imposed axial pre-stretches. The curve showing $d F / d p \sim 0$ corresponds, then, to that of the in vivo axial pre-stretch. This network of curves is given in Fig. 4 and it is derived from it that the in vivo axial pre-stretch of our model vein is about 1.5.

\subsection{Influence of vein constitutive properties}

The influence of the tissue anisotropy was studied by varying the parameter $\beta$ responsible for the anisotropy of the response of this constitutive model. The corresponding results are shown in Fig. 5 where the tortuosity index is plotted as a function of $\beta$ for different imposed axial prestretches and different levels of internal pressure. At a first glance, it is observed that in certain conditions, the tortuosity index remains equal or quasi-equal to one, which means that the vein does not buckle. If we look at the results in details, it is firstly observed that the more the vein segment is pre-stretched, the lower its tortuosity index is, which means that the vessel is less tortuous or less prone to become tortuous. A second trend is clear: the larger $\beta$, the lower the tortuosity index, whatever the imposed axial pre-stretch. Remember that $\beta=45^{\circ}$ corresponds to an isotropic-like tissue, in the sense that its initial (low-strain) response is the same in circumferential and axial directions. Then, in other words, these results show that the more anisotropic the tissue, in the sense of circumferential stiffening, the more tortuous it is at a given pressure.

The influence of the tissue stiffness was also studied by simultaneously scaling the parameters $\mathrm{C}_{10}$ and $\mathrm{k}_{1}$ of the constitutive model. The corresponding results are shown in Figs. 6 and 7 where the tortuosity index and critical pressure are plotted as a function of the stiffness scaling factor on a semi-log plot. The main trend highlighted by these results is that an increase in stiffness tends to decrease the tortuosity index of the vessel at a given pressure (Fig. 6). Fig. 7 shows a second very interesting trend. The effect of stiffness changes on the critical buckling pressure, i.e. the onset of tortuosity, depends on the imposed axial pre-stretch. When the vein is not, or weakly, axially pre-stretched, the critical pressure is found to decrease, or at least not increase, with increasing stiffness. On the contrary, from an axial pre-stretch of 1.4, the critical pressure significantly increases while the stiffness increases. 


\section{Discussion}

In the present paper a FE model was developed in order to study the buckling and induced tortuosity of vein segments within the context of diseased vein and associated tortuosity. The model was defined and calibrated based on a previous experimental work which was also used for the purpose of validation. While acceptable in the range of 1.2 to 1.5 of axial pre-stretch, the model seems to over-estimate the experimental critical buckling pressure especially at very large or very low pre-stretch values. A first reason for this is that the numerical approach intrinsically assumes a perfect geometry and homogeneity of the vessel, hence it is expected that critical buckling pressures are larger than the experimental ones. Indeed, imperfections in experimental specimens are known to reduce the critical buckling loads. These differences may also arise from the choice of the initial geometry of the vein segment. Indeed this geometry was chosen arbitrarily in the mid-range of the experimental geometries referred to above and the constitutive parameters were identified based on average Fung-type parameters previously fitted by Lee and Han (2010). There is no guarantee that the average constitutive parameters would correspond to the behavior of the average geometry vein segment, especially as it was shown earlier by Han (2008) that the influence of the length to radius ratio on the critical pressure is significant. Moreover, the experimental inflation-extension tests from which the model was identified provided few data points in the range 1 to 1.6 of axial pre-stretch (most of the recorded data were above), therefore the reliability of the constitutive parameters in this range might be improved.

Nevertheless, the trends observed experimentally are properly recovered by the model, which makes it suitable for the purpose of analyzing the influence of constitutive behavior evolutions such as those occurring during tissue remodeling and aging, which are also often associated with disorders of axial pre-stretch (Horny et al., 2011).

It was shown thanks to this model that the anisotropy of the tissue strongly affects the level of tortuosity of the vein segment at a given pressure. When the behavior of the tissue tends towards more circumferentially-oriented stiffness, the tortuosity or likelihood to buckle increases significantly under a given pressure. Also, it was found that the tortuosity index is influenced by the stiffness of the wall but, more interestingly, the influence of the stiffness on the critical buckling pressure depends on the axial pre-stretch of the vessel. At low axial prestretches, vessel stiffening tends to slightly decrease the critical pressure or has no effect on it, which means that the vessel is more likely or at least as likely to become tortuous. On the contrary, at higher axial pre-stretches, the effect of stiffening is to increase the critical pressure. 
Data regarding the risk of mechanical buckling of veins and its biomechanical aspects are scarce in the literature. Many clinical studies were focused on tortuosity of vessels itself and studied its possible clinical causes and consequences, especially for arteries. For instance, Smedby and Bergstrand (1996) focused on the relationship between femoral atherosclerosis and tortuosity, and concluded that tortuosity was not a consequence of atherosclerosis but rather a contributor to its development. Closer to mechanics, in their study on rabbit carotid arteries, Jackson et al. (2005) concluded that substantial axial pre-stretch is necessary to sustain the morphological stability of arteries, and that a reduction in this pre-stretch results in arterial tortuosity attributable to an aberrant biological remodeling activity. This finding is in total agreement with the results computed with the FE model developed in this study regarding the influence of axial pre-stretch. In addition, it raises the hypothesis that biological disorders lead to the development of tortuosity.

With regards to biomechanics and possible underlying mechanisms for vessel tortuosity, the group of H-C Han has been probably the most important publishing group. They studied vessel mechanical buckling by focusing on both arteries and veins, and by developing several analytical models as well as experimental studies (Han, 2011; Lee and Han, 2010; Liu and Han, 2012; Martinez et al., 2010). Part of this work motived the present study and was used in it. These analytical models were appropriate to describe buckling of vessels under pressure and study several parameters influencing the critical buckling pressure such as axial pre-stretch, length to diameter ratio, tapering of the vessel or surrounding tissue stiffness. For the purpose of validation, it was found that the present FE model is in agreement with the corresponding experimental data. Qualitatively the results presented in this paper are also in agreement with the previous theoretical works, though additional factors were focused on (influence of anisotropy and stiffness of the wall).

The present work constitutes an interesting contribution towards the comprehension of tortuosity formation. Indeed, Travers et al. (1996) observed that varicose saphenous veins contained significantly higher amounts of collagen in all layers of the vein wall and that these collagen fibers were seen to invade and break up regular muscle layers of the media in varicosis. In addition, several other biological changes occur in varicose veins (Naoum et al., 2007; Oklu et al., 2012; Urbanek et al., 2004; Woodside et al., 2003), which are very likely to affect the mechanical behavior of the wall. In an experimental mechanical testing study, McGilvray et al. (2010) found a marked stiffening in altered (post-phlebitic) venous walls compared to healthy ones. These findings, combined with our results, support the hypothesis that the changes accompanying venous diseases may promote tortuosity through buckling mechanisms. 
Following this hypothesis, it is also interesting to notice that external compression which is the usual recommended treatment for varicose veins will have positive effects on the buckling phenomenon. Its primary mechanism of action is to reduce the transmural pressure (Bergan, 2007). In the present FE model, a reduction of the transmural pressure would directly reduce the level of tortuosity or induce a proportional increase of the critical buckling pressure, thereby preventing from tortuosity onset and confirming the interest of such treatment. However in real leg geometries, Rohan et al. (2012) showed that the distribution of pressure in the vicinity of a varicose vein is not homogeneous. For this reason, the evaluation of elastic compression effects on tortuosity onset should be investigated more carefully by applying the actual pressure field around the vein segment.

Hence, this work motivates to pursue the modeling of the mechanism of buckling-induced tortuosity formation in more realistic conditions. In the long term, such a model will be used in complex in vivo configurations to better understand the pathology of vein tortuosity, the associated mechanisms and the effects of the treatment by elastic compression (Partsch et al., 2010; Rohan et al., 2012). Models including the surrounding materials and 3D leg geometry (Dubuis et al., 2011) will be developed to evaluate the potential of tortuosity forming in vivo through this mechanism and a clinical validation will be performed based on the identification of in vivo material properties of varicose veins. It is expected that this kind of model can be used to better understand the biomechanical aspects of this problem and, for instance, of the treatment by elastic compression. Also, it can be imagined that a constitutive model of the vein wall is developed including remodeling processes with the aim to predict the evolution of venous pathologies like varicosis.

\section{Conclusion}

In the present work, a Finite Element model, fairly simple for the moment, was introduced to validate the ability of a FE model to numerically simulate vein buckling and the onset of tortuosity. The model showed that the tortuosity of veins is increased when their axial prestretch decreases and when their behavior becomes more anisotropic (towards more circumferentially-oriented stiffness). It was also found that an increase in stiffness is beneficial with regards to the level of tortuosity.but only when the vein is axially pre-stretched by more than 1.2. In contrast, it was shown that the same increase in stiffness is detrimental, i.e. the critical pressure decreases, or has no effect when the vein segment is little or not pre-stretched. These results provide additional arguments supporting the hypothesis of tortuosity being the consequence of biologically-induced changes in the varicose vein wall. This model will be 
introduced in a 3D leg numerical model to study the phenomenon of vein tortuosity and elastic compression effects in more realistic conditions. A clinical validation is being developed based on the identification of in vivo material properties of varicose veins.

\section{References}

Attinger, E.0., 1969. Wall properties of veins. IEEE transactions on bio-medical engineering 16, 253-61.

Bergan, J. (Ed.), 2007. The vein book. Elsevier Academic Press.

Brossollet, L.J., Vito, R.P., 1995. An alternate formulation of blood vessel mechanics and the meaning of the in vivo property. Journal of biomechanics $28,679-687$.

Caggiati, A., Rosi, C., Heyn, R., Franceschini, M., Acconcia, M.C., 2006. Age-related variations of varicose veins anatomy. Journal of vascular surgery $44,1291-5$.

Carta, L., Wagenseil, J.E., Knutsen, R.H., Mariko, B., Faury, G., Davis, E.C., Starcher, B., Mecham, R.P., Ramirez, F., 2009. Discrete Contributions of Elastic Fiber Components to Arterial Development and Mechanical Compliance. Arteriosclerosis, Thrombosis, and Vascular Biology 29, 2083-2089.

Dubuis, L., Avril, S., Debayle, J., Badel, P., 2011. Identification of the material parameters of soft tissues in the compressed leg. Computer Methods in Biomechanics and Biomedical Engineering 15, 3-11.

Dye, W.W., Gleason, R.L., Wilson, E., Humphrey, J.D., 2007. Altered biomechanical properties of carotid arteries in two mouse models of muscular dystrophy. Journal of applied physiology (Bethesda, Md. : 1985) 103, 664-72.

Guo, X., Liu, Y., Kassab, G.S., 2012. Diameter-dependent axial prestretch of porcine coronary arteries and veins. Journal of applied physiology 112, 982-989.

Han, H.-C., 2008. Nonlinear buckling of blood vessels: a theoretical study. Journal of biomechanics 41, 2708-13.

Han, H.-C., 2011. Determination of the critical buckling pressure of blood vessels using the energy approach. Annals of biomedical engineering 39, 1032-40.

Holzapfel, G.A., Gasser, T.C., Ogden, R.W., 2000. A New Constitutive Framework for Arterial Wall Mechanics and a Comparative Study of Material Models. Journal of Elasticity 61, 1-48.

Holzapfel, G.A., Gasser, T.C., Ogden, R.W., 2004. Comparison of a Multi-Layer Structural Model for Arterial Walls With a Fung-Type Model, and Issues of Material Stability. Journal of Biomechanical Engineering 126, 264-275.

Horny, L., Adamek, T., Gultova, E., Zitny, R., Vesely, J., Chlup, H., Konvickova, S., 2011. Correlations between age, prestrain, diameter and atherosclerosis in the male abdominal aorta. Journal of the mechanical behavior of biomedical materials 4, 2128-32. 
Humphrey, J.D., Kang, T., Sakarda, P., Anjanappa, M., 1993. Computer-Aided Vascular Experimentation: A New Electromechanical Test System. Annals of biomedical engineering $21,33-43$.

Jackson, Z.S., Dajnowiec, D., Gotlieb, A.I., Langille, B.L., 2005. Partial off-loading of longitudinal tension induces arterial tortuosity. Arteriosclerosis, thrombosis, and vascular biology 25, 957-62.

Kim, J., Kang, Y., Choi, H., Hwang, S., Kang, B., 2002. Comparison of Implicit and Explicit FiniteElement Methods for the Hydroforming Process of an Automobile Lower Arm. International journal of advanced manufacturing technology 20, 407-413.

Lee, A.Y., Han, H.-C., 2010. A Nonlinear Thin-Wall Model for Vein Buckling. Cardiovascular engineering 1, 282-289.

Lim, C.S., Davies, A.H., 2009. Pathogenesis of primary varicose veins. British Journal of Surgery 96, 1231-1242.

Liu, Q., Han, H.-C., 2012. Mechanical buckling of artery under pulsatile pressure. Journal of biomechanics 45, 1192-8.

Martinez, R., Fierro, C. a, Shireman, P.K., Han, H.-C., 2010. Mechanical buckling of veins under internal pressure. Annals of biomedical engineering 38, 1345-53.

McGilvray, K.C., Sarkar, R., Nguyen, K., Puttlitz, C.M., 2010. A biomechanical analysis of venous tissue in its normal and post-phlebitic conditions. Journal of biomechanics 43, 2941-7.

Naoum, J.J., Hunter, G.C., Woodside, K.J., Chen, C., 2007. Current advances in the pathogenesis of varicose veins. The Journal of surgical research 141, 311-6.

Oklu, R., Habito, R., Mayr, M., Deipolyi, A.R., Albadawi, H., Hesketh, R., Walker, T.G., Linskey, K.R., Long, C. a, Wicky, S., Stoughton, J., Watkins, M.T., 2012. Pathogenesis of varicose veins. Journal of vascular and interventional radiology 23, 33-9.

Rohan, C..-Y., Badel, P., Lun, B., Rastel, D., Avril, S., 2012. Biomechanical response of varicose veins to elastic compression: A numerical study. Journal of Biomechanics in press.

Smedby, O., Bergstrand, L., 1996. Tortuosity and Atherosclerosis in the Femoral Artery: What is Cause and What is Effect? Annals of biomedical engineering 24, 474-480.

Travers, J.P., Brookes, C.E., Evans, J., Baker, D.M., Kent, C., Makin, G.S., Mayhew, T.M., 1996. Assessment of wall structure and composition of varicose veins with reference to collagen, elastin and smooth muscle content. European journal of vascular and endovascular surgery $11,230-7$.

Urbanek, T., Skop, B., Wiaderkiewicz, R., Wilczok, T., Ziaja, K., Lebda-Wyborny, T., Pawlicki, K., 2004. Smooth muscle cell apoptosis in primary varicose veins. European journal of vascular and endovascular surgery $28,600-11$.

Van Loon, P., Klip, W., Bradley, E., 1977. Length-force and volume-pressure relationships of arteries. Biorheology 14, 181-201. 
Wagenseil, J.E., Nerurkar, N.L., Knutsen, R.H., Okamoto, R.J., Li, D.Y., Mecham, R.P., 2005. Effects of elastin haploinsufficiency on the mechanical behavior of mouse arteries. American journal of physiology. Heart and circulatory physiology 289, H1209-17.

Weizsacker, H., Pinto, J., 1988. Isotropy and anisotropy of arterial wall. Journal of Biomechanics 21, 477-487.

Weizsäcker, H.W., 1988. Passive elastic properties of the rat abdominal vena cava. European Joumal of Physiology 412, 147-154.

Woodside, K.J., Hu, M., Burke, A., Murakami, M., Pounds, L.L., Killewich, L. a, Daller, J. a, Hunter, G.C., 2003. Morphologic characteristics of varicose veins: possible role of metalloproteinases. Journal of Vascular Surgery 38, 162-169.

Xiao, Y., Huang, Z., Yin, H., Lin, Y., Wang, S., 2009. In vitro differences between smooth muscle cells derived from varicose veins and normal veins. Journal of vascular surgery 50, 114954. 


\section{List of figures}

Figure 1: Schematic of a vein as considered with the Holzapfel constitutive model ; fiber angle $\beta$ is defined with respect to the circumferential direction.

Figure 2: Response of the reference model in terms of tortuosity index as a function of pressure and axial pre-stretch. Illustrations of the deformed geometry at pressures of $0,10,12.5$ and 100 mmHg are added to the graph.

Figure 3: Critical buckling pressure computed using the reference model compared to the experimental values.

Figure 4: Numerical experiment of pressurization under imposed axial pre-stretch using the reference model parameters. The results show the axial force as a function of the pressure.

Figure 5: Influence of the anisotropy of the constitutive model. The tortuosity index is plotted vs. the parameter $\beta$ for an internal pressure of (A) $20 \mathrm{mmHg}$, (B) $45 \mathrm{mmHg}$ and (C) $70 \mathrm{mmHg}$.

Figure 6: Influence of the stiffness of the constitutive model. The tortuosity index is plotted vs. the stiffness ratio for an internal pressure of (A) $20 \mathrm{mmHg}$, (B) $45 \mathrm{mmHg}$ and (C) $70 \mathrm{mmHg}$.

Figure 7: Influence of the stiffness of the constitutive model on the critical buckling pressure. 


\section{List of tables}

Table 1: Material parameters determined by inverse identification based on the data provided by Martinez et al. (2010).

\begin{tabular}{|l|l|}
\hline $\mathrm{C}(\mathrm{kPa})$ & 1 \\
\hline $\mathrm{k} 1(\mathrm{kPa})$ & 5.6 \\
\hline $\mathrm{k} 2$ & 2.9 \\
\hline$\beta\left(^{\circ}\right)$ & 25 \\
\hline
\end{tabular}

